\title{
PERSISTENCE AND GLOBAL ASYMPTOTIC STABILITY OF SINGLE SPECIES DISPERSAL MODELS WITH STAGE STRUCTURE
}

\author{
BY
}

\author{
H. I. FREEDMAN*AND J. H. WU ${ }^{\dagger}$
}

University of Alberta, Edmonton, Alberta, Canada T6G 2G1

\begin{abstract}
A system of retarded functional differential equations is proposed as a model of single-species population growth with dispersal in a multi-patch environment where individual members of the population have a life history that takes them through two stages, immature and mature. The persistence of the system as well as the existence and global asymptotic stability of a positive equilibrium is proved by using the monotone dynamical systems theory due to Hirsch and Smith, and a convergence theorem established in this paper for nonautonomous retarded equations by using limiting equations theory.
\end{abstract}

1. Introduction. The effect of environment change in the growth and diffusion of a species in a heterogeneous habitat is a subject of considerable interest in the ecological literature. The theoretical study of this subject was pioneered by Skellam [35] and detailed bibliographies can be found in the work of Levin [29]. To study such a habitat effect, one technique is to divide the habitat into two or more homogeneous patches connected by dispersion. Much research has been devoted to the mathematical analysis of model equations for the growth of single-species population dispersing among patches in a heterogeneous environment. For details we refer to $[5,6,9-15$, $23,25,31,34,35,37,38,41]$.

On the other hand, the description of the age structure of the population in the long run is also an interesting problem in population dynamics. Many models have been proposed and analyzed concerning single-species population growth with various stages of life history. Bibliographies can be found in [1, 2, 4, 17, 18, 28, 39-41].

It is the main purpose of this paper to propose and analyze a model of singlespecies population growth dispersing in a multi-patch environment, where individual

Received June 6, 1990.

* Research partially supported by the Natural Sciences and Engineering Research Council of Canada, Grant No. NSERC A4823.

${ }^{\dagger}$ Research partially supported by the Gordin Kaplan Postdoctoral Fellowship and by the Central Research Fund of the University of Alberta. Present address: Department of Mathematics, York University, North York, Ontario, Canada M3J 1P3. 
members of the population have a life history that takes them through two stages, immature and mature. Under the assumption that the dispersion of population between patches is symmetric, the subsystem for the mature population in our model is a cooperative and irreducible system of retarded functional differential equations with a discrete time delay representing the time from birth to maturity. By using the monotone dynamical systems theory of Hirsch [24] and Smith [36], we will show the persistence of the subsystem for the mature population and the existence as well as the global asymptotic stability of a positive equilibrium state. The subsystem for the immature population is a system of ordinary differential equations with a perturbation term representing the net flow of the birth from the mature population into immature population and the removal rate from immature population to mature population. We will show that this subsystem has a positive equilibrium state which is globally asymptotically stable.

We will also investigate the global convergence problem in the case of nonsymmetric dispersal of population between different patches by using a convergence theorem via limiting equations for general nonautonomous "regular" and "precompact" functional differential equations, representing an extension of a result due to Artstein [3] for nonautonomous ordinary differential equations to retarded equations.

We should mention that the global stability of the positive equilibrium state has been proved by Aiello and Freedman [1] for a model of a single-species population with stage structures where the population is assumed to be distributed over a homogeneous environment. For other related results, we refer to [1,2] and references therein. Our results in this paper show that, as far as our model is concerned, the heterogeneity of the environment may change the size of the positive equilibrium state but cannot change its global asymptotic stability.

This paper is organized as follows. In Sec. 2, we develop our model and present some standing assumptions. In Sec. 3, we consider the global dynamics of both mature populations and immature populations in the case of symmetric dispersion of the population between patches. Section 4 contains a global convergence result in the case of nonsymmetric dispersion of the population between two patches. In Sec. 5 , we briefly indicate how the approach employed in Sects. 3 and 4 can be effectively combined to establish the global asymptotic stability of a positive equilibrium state in a general multipatch environment with possible nonsymmetric dispersion.

2. Model equations. We suppose that the system is composed of $n$ patches connected by dispersion and occupied by a single species. Let $I_{i}(t)$ and $M_{i}(t)$ denote the concentration of immature and mature populations in the $i$ th patch, $i=1,2, \ldots, n$. To derive our model equations, we make the following assumptions.

$(\mathrm{H} 1)$ : The birth rate into the immature population in the $i$ th patch is proportional to the existing mature population with proportionality constant $\alpha_{i}>0, i=$ $1, \ldots, n$.

$(\mathrm{H} 2)$ : The death rate of the immature population in the $i$ th patch is proportional to the existing immature population with proportionality constant $\gamma_{i}>0$, $i=1, \ldots, n$. 
(H3): The death rate of the mature population in the $i$ th patch is of a logistic nature, i.e., proportional to the square of the population with proportionality constant $\beta_{i}>0, i=1, \ldots, n$.

(H4): The length of time from birth to maturity is a constant $\tau>0$, which is uniform for each individual in all patches.

(H5): Those immature individuals born at time $t-\tau$ and surviving to time $t$ exit from the immature population and enter into the mature population.

(H6): The net exchange of mature and immature populations from the $j$ th patch to the $i$ th patch is proportional to the difference of the concentrations $M_{j}(t)-$ $M_{i}(t)$ and $I_{j}(t)-I_{i}(t)$, respectively, with proportionality constants $D_{j i} \geq 0$ and $\delta_{j i} \geq 0, i \neq j, i, j=1, \ldots, n$.

Under the above assumptions, we propose a model to describe the growth of a single-species population dispersing in an $n$ patch environment where individual members of the population have a two-stage structure as follows

$$
\begin{gathered}
\frac{d}{d t} I_{i}(t)=-\gamma_{i} I_{i}(t)+\sum_{j \neq i} \delta_{j i}\left[I_{j}(t)-I_{i}(t)\right]+\alpha_{i} M_{i}(t)-x_{i}(t, t-\tau), \\
\frac{d}{d t} M_{i}(t)=-\beta_{i} M_{i}^{2}(t)+\sum_{j \neq i} D_{j i}\left[M_{j}(t)-M_{i}(t)\right]+x_{i}(t, t-\tau), \\
t \geq 0, \quad i=1, \ldots, n,
\end{gathered}
$$

where for any $s, t-\tau \leq s \leq t, x_{i}(s, t-\tau)$ denotes the growth rate at the instant $s$ of the immature population in the $i$ th patch born at the instant $t-\tau$.

Obviously,

$$
x_{i}(t-\tau, t-\tau)=\alpha_{i} M_{i}(t-\tau) .
$$

To derive an explicit formula for $x_{i}(s, t-\tau)$ in terms of $M_{j}(t), j=1, \ldots, n$, we denote by $y_{i}(s, t-\tau), t-\tau \leq s \leq t$, the total immature population in the $i$ th patch born at the instant $t-\tau$. Then $\frac{\partial}{\partial s} y_{i}(s, t-\tau)=x_{i}(s, t-\tau)$ and we have the following relation

$$
x_{i}(s, t-\tau)=-\gamma_{i} y_{i}(s, t-\tau)+\sum_{j \neq i} \delta_{j i}\left[y_{j}(s, t-\tau)-y_{i}(s, t-\tau)\right]
$$

from which it follows that

$$
\begin{array}{r}
\frac{\partial}{\partial s} y_{i}(s, t-\tau)=-\gamma_{i} y_{i}(s, t-\tau)+\sum_{j \neq i} \delta_{j i}\left[y_{j}(s, t-\tau)-y_{i}(s, t-\tau)\right], \\
t-\tau \leq s \leq t .
\end{array}
$$

Let $A=\left(a_{i j}\right)$ with

$$
a_{i j}=\delta_{j i} \text { for } i, j=1, \ldots, n, i \neq j \text {, }
$$

and

$$
a_{i i}=-\gamma_{i}-\sum_{j \neq i} \delta_{j i}, \quad i=1, \ldots, n
$$


Then $a_{i j} \geq 0$ for $i \neq j$, and $\sum_{j=1}^{n} a_{i j}=-\gamma_{i}<0$ for $i=1, \ldots, n$. Therefore $A^{-1}$ exists and for any $t \geq 0, e^{A t}$ is a matrix whose entries are nonnegative. Evidently, (2.3) can be rewritten as

$$
\frac{\partial}{\partial s}\left(\begin{array}{c}
y_{1}(s, t-\tau) \\
\vdots \\
y_{n}(s, t-\tau)
\end{array}\right)=A\left(\begin{array}{c}
y_{1}(s, t-\tau) \\
\vdots \\
y_{n}(s, t-\tau)
\end{array}\right)
$$

from which it follows that

and thus

$$
\left(\begin{array}{c}
y_{1}(s, t-\tau) \\
\vdots \\
y_{n}(s, t-\tau)
\end{array}\right)=e^{A(s-t+\tau)}\left(\begin{array}{c}
y_{1}(t-\tau, t-\tau) \\
\vdots \\
y_{n}(t-\tau, t-\tau)
\end{array}\right)
$$

$$
\frac{\partial}{\partial s}\left(\begin{array}{c}
y_{1}(s, t-\tau) \\
\vdots \\
y_{n}(s, t-\tau)
\end{array}\right)=A e^{A(s-t+\tau)}\left(\begin{array}{c}
y_{1}(t-\tau, t-\tau) \\
\vdots \\
y_{n}(t-\tau, t-\tau)
\end{array}\right) .
$$

Substituting (2.2) into (2.4), we get

$$
\left(\begin{array}{c}
\alpha_{1} M_{1}(t-\tau) \\
\vdots \\
\alpha_{n} M_{n}(t-\tau)
\end{array}\right)=A\left(\begin{array}{c}
y_{1}(t-\tau, t-\tau) \\
\vdots \\
y_{n}(t-\tau, t-\tau)
\end{array}\right)
$$

Consequently,

$$
\left(\begin{array}{c}
y_{1}(t-\tau, t-\tau) \\
\vdots \\
y_{n}(t-\tau, t-\tau)
\end{array}\right)=A^{-1}\left(\begin{array}{c}
\alpha_{1} M_{1}(t-\tau) \\
\vdots \\
\alpha_{n} M_{n}(t-\tau)
\end{array}\right)
$$

and

$$
\frac{\partial}{\partial s}\left(\begin{array}{c}
y_{1}(s, t-\tau) \\
\vdots \\
y_{n}(s, t-\tau)
\end{array}\right)=A e^{A(s-t+\tau)} A^{-1}\left(\begin{array}{c}
\alpha_{1} M_{1}(t-\tau) \\
\vdots \\
\alpha_{n} M_{n}(t-\tau)
\end{array}\right)=e^{A(s-t+\tau)}\left(\begin{array}{c}
\alpha_{1} M_{1}(t-\tau) \\
\vdots \\
\alpha_{n} M_{n}(t-\tau)
\end{array}\right) .
$$

Therefore

$$
\left(\begin{array}{c}
x_{1}(t, t-\tau) \\
\vdots \\
x_{n}(t, t-\tau)
\end{array}\right)=\left.\frac{\partial}{\partial s}\left(\begin{array}{c}
y_{1}(s, t-\tau) \\
\vdots \\
y_{n}(s, t-\tau)
\end{array}\right)\right|_{s=t}=e^{A \tau}\left(\begin{array}{c}
\alpha_{1} M_{1}(t-\tau) \\
\vdots \\
\alpha_{n} M_{n}(t-\tau)
\end{array}\right) .
$$

Let $e^{A \tau}=\left(b_{i j}\right)$. Then $b_{i j} \geq 0$ for $i, j=1, \ldots, n$ and

$$
x_{i}(t, t-\tau)=\sum_{j=1}^{n} b_{i j} \alpha_{j} M_{j}(t-\tau)
$$

from which our model equation (2.1) can be reduced to the following form $\left\{\begin{array}{l}\frac{d}{d t} I_{i}(t)=-\gamma_{i} I_{i}(t)+\sum_{j \neq i} \delta_{j i}\left[I_{j}(t)-I_{i}(t)\right]+\alpha_{i} M_{i}(t)-\sum_{j=1}^{n} b_{i j} \alpha_{j} M_{j}(t-\tau), \\ \frac{d}{d t} M_{i}(t)=-\beta_{i} M_{i}^{2}(t)+\sum_{j \neq i} D_{j i}\left[M_{j}(t)-M_{i}(t)\right]+\sum_{j=1}^{n} b_{i j} \alpha_{j} M_{j}(t-\tau),\end{array}\right.$ for $t \geq 0$ and $l=1, \ldots, n$. 
To specify a solution of the model equation (2.5), we assume that the distribution of the mature population over the initial period $-\tau \leq t \leq 0$ is given as follows

$$
M_{i}(\theta)=\varphi_{i}(\theta), \quad-\tau \leq \theta \leq 0, i=1,2, \ldots, n,
$$

and the immature population at $t=0$ is given as follows

$$
I_{i}(0)=I_{i 0}, \quad i=1,2, \ldots, n,
$$

where $\varphi_{i}(\theta)$ is a nonnegative continuous function on $[-\tau, 0]$ and $I_{i 0}$ is a given nonnegative constant, $i=1, \ldots, n$.

3. Persistence and global convergence in the case of symmetric dispersal. In this section, we consider the global dynamics of our model equation (2.5) under the following assumption concerning the dispersion between different patches.

(H7): The dispersion matrices $D=\left(D_{i j}\right)$ and $\Delta=\left(\delta_{i j}\right)$, where $\delta_{i i}=D_{i i}=0$, $i=1, \ldots, n$ are irreducible.

We begin by considering the subsystem for the mature populations

$$
\frac{d}{d t} M_{i}(t)=-\beta_{i} M_{i}^{2}(t)+\sum_{j \neq i} D_{j i}\left[M_{j}(t)-M_{i}(t)\right]+\sum_{j=1}^{n} b_{i j} \alpha_{j} M_{j}(t-\tau)
$$

where $t \geq 0, i=1, \ldots, n$. Let $C_{n}=C\left([-\tau, 0] ; R^{n}\right)$ and $C_{n}^{+}=\left\{\varphi \in C_{n} ; \varphi=\right.$ $\left(\varphi_{1}, \ldots, \varphi_{n}\right), \varphi_{i}(\theta) \geq 0$ for $i=1, \ldots, n$ and $\left.\theta \in[-\tau, 0]\right\}$. Define $F=\left(F_{1}, \ldots\right.$, $\left.F_{n}\right): C_{n} \rightarrow R^{n}$ by

$$
F_{i}(\varphi)=-\beta_{i} \varphi_{i}^{2}(0)+\sum_{j \neq i} D_{j i}\left[\varphi_{j}(0)-\varphi_{i}(0)\right]+\sum_{j=1}^{n} b_{i j} \alpha_{j} \varphi_{j}(-\tau), \quad i=1, \ldots, n,
$$

where $\varphi \in C_{n}$. It is easy to verify the following quasimonotonicity condition: for any $\varphi \in C_{n}^{+}$, if $\varphi_{i}(0)=0$, then $F_{i}(\varphi) \geq 0$. Therefore, by Corollary 2.1 of Smith [36], $C_{n}^{+}$is positively invariant, i.e., for any $\varphi \in C_{n}^{+}, M_{i}(t, \varphi) \geq 0$ for $t \geq 0$ and $i=1, \ldots, n$, where $M(t, \varphi)=\left(M_{1}(t, \varphi), \ldots, M_{n}(t, \varphi)\right)$ is the unique solution of (3.1) satisfying $M_{i}(\theta, \varphi)=\varphi_{i}(\theta)$ for $\theta \in[-\tau, 0]$ and $i=1, \ldots, n$.

The following result indicates that all solutions of system (3.1) are bounded.

Lemma 3.1. For any $\varphi \in C_{n}^{+}$, we have

$$
0 \leq M_{i}(t, \varphi) \leq L(\varphi), \quad t \geq 0, i=1, \ldots, n
$$

where

Proof. Let

$$
L(\varphi)=\max \left\{\max _{-\tau \leq \theta \leq 0} \max _{i=1, \ldots, n} \varphi_{i}(\theta), \max _{i=1, \ldots, n} \frac{\sum_{j=1}^{n} b_{i j} \alpha_{j}}{\beta_{i}}+1\right\} .
$$

$$
V(t)=\max \left\{\max _{-\tau \leq \theta \leq t} \max _{i=1, \ldots, n} M_{i}(\theta, \varphi), \max _{i=1, \ldots, n} \frac{\sum_{j=1}^{n} b_{i j} \alpha_{j}}{\beta_{i}}+1\right\} .
$$

For any given $t \geq 0$, if $\max _{i=1, \ldots, n} M_{i}(t, \varphi)<V(t)$, then clearly $D^{+} V(t)=0$, where

$$
D^{+} V(t)=\limsup _{h \rightarrow 0^{+}} \frac{V(t+h)-V(t)}{h} .
$$


If $\max _{1, \ldots, n} M_{i}(t, \varphi)=V(t)$, then for any $j \notin J:=\left\{k: M_{k}(t, \varphi)=V(t)\right\}$, $M_{j}(t+h, \varphi)<M_{k}(t+h, \varphi)$ for any $k \in J$ and sufficiently small $h \geq 0$. Select a sequence $h_{m} \rightarrow 0^{+}$such that

$$
D^{+} V(t)=\lim _{m \rightarrow \infty} \frac{V\left(t+h_{m}\right)-V(t)}{h_{m}} .
$$

Then two cases may occur:

CASE 1 . There are infinitely many $h_{m}$ 's such that $V\left(t+h_{m}\right) \leq V(t)$.

In this case, it is clear that $D^{+} V(t) \leq 0$.

CASE 2. There are only finitely many $h_{m}$ 's such that $V\left(t+h_{m}\right) \leq V(t)$.

In this case, there exist a $k \in J$ and a sequence $\left\{h_{m}^{*}\right\}$ with $0<h_{m}^{*} \leq h_{m}$ such that $V\left(t+h_{m}\right)=M_{k}\left(t+h_{m}^{*}, \varphi\right)$. Therefore

$$
D^{+} V(t)=\lim _{m \rightarrow \infty} \frac{M_{k}\left(t+h_{m}^{*}, \varphi\right)-M_{k}(t, \varphi)}{h_{m}} .
$$

Note that

$$
\begin{aligned}
& \lim _{m \rightarrow \infty} \frac{M_{k}\left(t+h_{m}^{*}, \varphi\right)-M_{k}(t, \varphi)}{h_{m}^{*}} \\
& \quad=\frac{d}{d t} M_{k}(t, \varphi) \\
& \quad=-\beta_{k} M_{k}^{2}(t, \varphi)+\sum_{j \neq k} D_{j k}\left[M_{j}(t, \varphi)-M_{k}(t, \varphi)\right]+\sum_{j=1}^{n} b_{k j} \alpha_{j} M_{j}(t-\tau, \varphi) . \\
& \quad \leq-\beta_{k} M_{k}^{2}(t, \varphi)+\sum_{j=1}^{n} b_{k j} \alpha_{j} M_{j}(t-\tau, \varphi) \\
& \quad \leq\left[\frac{\sum_{j=1}^{n} b_{k j} \alpha_{j}}{\beta_{k}}-M_{k}(t, \varphi)\right] \beta_{k} M_{k}(t, \varphi)<0 .
\end{aligned}
$$

Therefore, $M_{k}\left(t+h_{m}^{*}, \varphi\right)<M_{k}(t, \varphi)$ for sufficiently large $m$, from which result together with (3.2) it follows that $D^{+} V(t) \leq 0$.

Therefore for any $t \geq 0, D^{+} V(t) \leq 0$ holds. By the well-known result of differential inequalities (see, e.g., [28]), we obtain $V(t) \leq V(0)$ for $t \geq 0$, from which our conclusion follows. This completes the proof.

The following result shows a dissipative property of system (3.1).

Lemma 3.2. There exists a constant $B>0$ such that for any $\varphi \in C_{n}^{+}$, we have

$$
\limsup _{t \rightarrow \infty}\left[M_{1}(t, \varphi)+\cdots+M_{n}(t, \varphi)\right]<B .
$$

Proof. Let $\rho>1$ be a given constant and

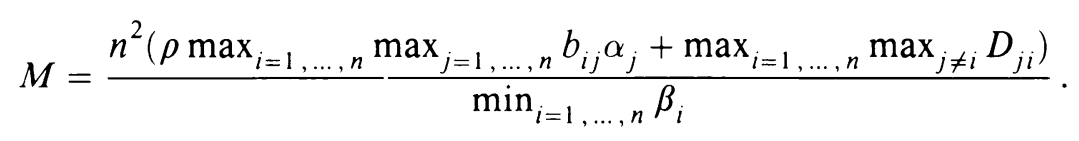


Define $M_{i}(t)=M_{i}(t, \varphi)$ and $W(t)=\sum_{i=1}^{n} M_{i}(t)$. Then

$$
\begin{aligned}
\dot{W}(t)= & \sum_{i=1}^{n}\left[-\beta_{i} M_{i}^{2}(t)+\sum_{j \neq i} D_{j i}\left(M_{j}(t)-M_{i}(t)\right)+\sum_{j=1}^{n} b_{i j} \alpha_{j} M_{j}(t-\tau)\right] \\
\leq & -\left(\min _{i=1, \ldots, n} \beta_{i}\right) \sum_{i=1}^{n} M_{i}^{2}(t)+n\left(\max _{i=1, \ldots, n} \max _{j \neq i} D_{j i}\right) W(t) \\
& +n \max _{i=1, \ldots, n} \max _{j=1, \ldots, n} b_{i j} \alpha_{j} W(t-\tau) \\
\leq & -\frac{\min _{i=1, \ldots, n} \beta_{i}}{n} W^{2}(t)+n\left(\max _{i=1, \ldots, n} \max _{j \neq i} D_{j i}\right) W(t) \\
& +n \max _{i=1, \ldots, n} \max _{j=1, \ldots, n} b_{i j} \alpha_{j} W(t-\tau) .
\end{aligned}
$$

Therefore, if $W(t-\tau) \leq \rho W(t)$ and $W(t)>M$, then

$$
\begin{aligned}
\dot{W}(t) \leq & {\left[\frac{n^{2}\left(\max _{i=1, \ldots, n} \max _{j \neq i} D_{j i}+\rho \max _{i=1, \ldots, n} \max _{j=1, \ldots, n} b_{i j} \alpha_{j}\right)}{\min _{i=1, \ldots, n} \beta_{i}}-W(t)\right] } \\
& \times W(t) \frac{\min _{i=1, \ldots, n} \beta_{i}}{n}<0 .
\end{aligned}
$$

Therefore by the classical Liapunov-Razumikhin theorem for uniformly ultimate boundedness (see, e.g., [21]), there exists a constant $B>0$ uniformly for all $\varphi \in C_{n}^{+}$ such that $\lim _{t \rightarrow \infty} \sup \sum_{i=1}^{n} M_{i}(t, \varphi)<\beta$. This completes the proof.

By the general dissipative dynamical systems theory (see, e.g., [22]) system (3.1) has a globally compact attractor. We want to show that this attractor contains at least one positive equilibrium point. To provide a tool for the study of the existence and stability problem of equilibria, we briefly describe a result due to Smith [36].

Suppose $f: C_{n} \rightarrow R^{n}$ is continuously differentiable such that for any $\varphi \in C_{n}$ there exists one and only one solution of the following initial value problem

$$
\left\{\begin{array}{l}
\frac{d}{d t} x(t)=f\left(x_{t}\right), \quad t \geq 0, \\
x_{0}=\varphi
\end{array}\right.
$$

Let $x(t, \varphi)$ be the unique solution. We obtain a semiflow $\{T(t)\}_{t \geq 0}$ defined on $C_{n}$ as follows

$$
T(t) \phi=x_{t}(\phi) \text { for } t \geq 0 \text { and } \varphi \in C_{n} .
$$

Denote by $d f(\psi)$ the Frechlet derivative of $f$ at $\psi$. Then by the Riesz representation theorem we have the following standard representation

$$
d f(\psi) \phi=\sum_{j=1}^{n} \int_{-\tau}^{0} \phi_{j}(\theta) d \eta_{i j}(\psi, \theta),
$$

where for each $\psi \in C_{n}, \eta_{i j}(\psi, \cdot): R \rightarrow R$ satisfies $\eta_{i j}(\psi, \theta)=\eta_{i j}(\psi, 0)$ for $\theta \geq 0, \eta_{i j}(\psi, \theta)=0$ for $\theta \leq-\tau, \eta_{i j}(\psi, \cdot)$ is of bounded variation on $[-\tau, 0]$ and 
is continuous from the left on $[-\tau, 0], i, j=1, \ldots, n$. We say $f$ is cooperative and irreducible in $C_{n}^{+}$, if for any $\psi \in C_{n}^{+}$the following conditions are satisfied.

(i) For any $\phi \in C_{n}^{+}$with $\varphi_{i}(0)=0$, it follows that $\sum_{j=1}^{n} \int_{-\tau}^{0} \phi_{j}(\theta) d \eta_{i j}(\psi, \theta) \geq$ 0 .

(ii) The matrix $\left(d f(\psi) \hat{e}_{1}, \ldots, d f(\psi) \hat{e}_{n}\right)$ is irreducible, where $\left\{e_{1}, \ldots, e_{n}\right\}$ denotes the standard basis in $R^{n}$ and denotes the inclusion $R^{n} \rightarrow C_{n}$ by $x \rightarrow \hat{x}, \hat{x}_{i}(\theta)=x_{i}$ for $\theta \in[-\tau, 0]$ and $i=1, \ldots, n$.

(iii) For every $j$ there exists $i$ such that for all $\psi \in C_{n}^{+}$and all small positive $\varepsilon, \eta_{i j}(\psi,-\tau+\varepsilon)>0$.

In Smith [36] the following result is proved.

LEMMA 3.3. Let $f$ be cooperative and irreducible in $C_{n}^{+}$. Then the semiflow $\{T(t)\}_{t \geq 0}$ defined on $C_{n}^{+}$is monotone and eventually strongly monotone. That is,

(i) if $\psi-\phi \in C_{n}^{+}$, then $x_{t}(\psi)-x_{t}(\phi) \in C_{n}^{+}$for $t \geq 0$;

(ii) if $\psi-\phi \in C_{n}^{+} \backslash\{0\}$, then $x_{t}(\psi)-x_{t}(\phi) \in \operatorname{Int} C_{n}^{+}$for $t \geq(n+1) \tau$.

As an application of the above result, we obtain the following result concerning the persistence of system (3.1).

THEOREM 3.1. For any $\phi \in C_{n}^{+} \backslash\{0\}$, we have $\liminf _{t \rightarrow \infty} M_{i}(t, \varphi)>0$ for $i=$ $1, \ldots, n$.

Proof. It is clear that $F: C_{n} \rightarrow R^{n}$ is continuously differentiable. For any $\psi \in C_{n}^{+}$ and $\varphi \in C_{n}$, we have

$$
d F_{i}(\psi) \phi=-2 \beta_{i} \psi_{i}(0) \phi_{i}(0)+\sum_{j \neq i} D_{j i}\left[\phi_{j}(0)-\phi_{i}(0)\right]+\sum_{j=1}^{n} b_{i j} \alpha_{j} \phi_{j}(-\tau),
$$

where $i=1, \ldots, n$ and $d F(\psi)=\left(d F_{1}(\psi), \ldots, d F_{n}(\psi)\right)$. Therefore we have the following observations.

(i) $d F_{i}(\psi) \phi \geq 0$ if $\phi \in C_{n}^{+}$and $\phi_{i}(0)=0$.

(ii) The matrix

$$
\begin{aligned}
& \left(d F(\psi) \hat{e}_{1}, \ldots, d F(\psi) \hat{e}_{n}\right)=
\end{aligned}
$$

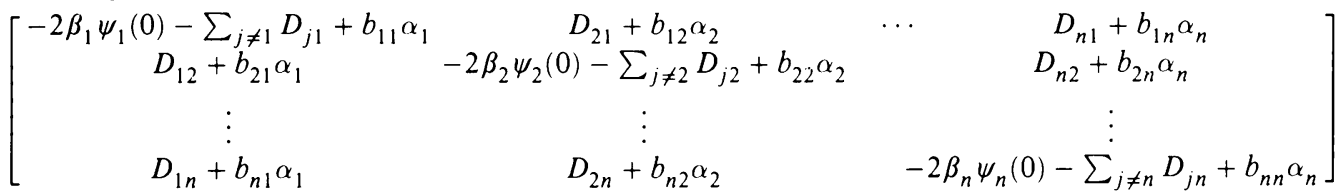

is irreducible, since by assumption $(\mathrm{H} 7)$, the diffusion matrix $D$ is irreducible and the matrix $e^{A \tau}=\left(b_{i j}\right)$ is positive.

Therefore, $F$ is cooperative and irreducible in $C_{n}^{+}$. By Lemma 3.3, we know that if $\phi \in C_{n}^{+} \backslash\{0\}$, then $M_{i}(t, \varphi)>0$ for $t \geq n \tau$ and $i=1, \ldots, n$. To prove the theorem, it suffices to verify that if $\varepsilon>0$ is sufficiently small, then the set $C_{n}^{+}(\varepsilon)=\left\{\varphi \in C_{n}^{+} ; \varphi_{i}(\theta) \geq \varepsilon\right.$ for $\theta \in[-\tau, 0]$ and $\left.i=1, \ldots, n\right\}$ is positively invariant, i.e., if $\varphi \in C_{n}^{+}(\varepsilon)$ then $M_{i}(t, \varphi) \geq \varepsilon$ for $t \geq 0$ and $i=1, \ldots, n$. This is 
true for any $\varepsilon$ with $0<\varepsilon<\min _{i=1, \ldots, n}\left(\sum_{j=1}^{n} b_{i j} \alpha_{j}\right) / \beta_{i}$, since for any $\varphi \in C_{n}^{+}(\varepsilon)$ if $\varphi_{i}(0)=\varepsilon$ for some $i$, then

$$
\begin{aligned}
F_{i}(\phi) & =-\beta_{i} \varepsilon^{2}+\sum_{j \neq i} D_{j i}\left[\varphi_{j}(0)-\phi_{i}(0)\right]+\sum_{j=1}^{n} b_{i j} \alpha_{j} \varphi_{j}(-\tau) \\
& \geq-\beta_{i} \varepsilon^{2}+\sum_{j \neq i} D_{j i}\left[\varphi_{j}(0)-\varepsilon\right]+\sum_{j=1}^{n} b_{i j} \alpha_{j} \varepsilon \\
& =\left(-\beta_{i} \varepsilon+\sum_{j=1}^{n} b_{i j} \alpha_{j}\right) \varepsilon \geq 0
\end{aligned}
$$

(see, e.g. [30]). The proof is then completed.

We will show that the persistence implies the existence, uniqueness, and global asymptotic stability of a positive equilibrium state. To show this, we need the following result due to Hirsch [24].

Lemma 3.4. Suppose $\widetilde{F}=\left(\widetilde{F}_{1}, \ldots, \widetilde{F}_{n}\right)$ is a $C^{1}$ vector field in $R^{n}$ satisfying the following conditions.

(i) The off-diagonal elements of the Jacobian $D \widetilde{F}(x)$ of $\widetilde{F}(x)$ are nonnegative for any $x \in R_{+}^{n}:=\left\{x \in R^{n}: x=\left(x_{1}, \ldots, x_{n}\right), x_{i} \geq 0, i=1, \ldots, n\right\}$.

(ii) $D \widetilde{F}(x)$ is irreducible for any $x \in R_{+}^{n}$.

(iii) $D \widetilde{F}(y)-D \widetilde{F}(x) \in R_{+}^{n \times n}$ for any $x, y \in R_{+}^{n}$ with $x-y \in R_{+}^{n}$.

(iv) All solutions in $R_{+}^{n}$ of the system $\dot{x}=\widetilde{F}(x)$ are bounded.

Then either trajectories in $R_{+}^{n}$ tend to the origin, or else there is a unique equilibrium $p \in \operatorname{Int} R_{+}^{n}$ and all trajectories in $R_{+}^{n} \backslash\{0\}$ tend to $p$.

THEOREM 3.2. System (3.1) has one and only one positive equilibrium. This positive equilibrium is globally asymptotically stable over $C_{n}^{+} \backslash\{0\}$.

Proof. According to the proof of Theorem 3.1, the set $C_{n}^{+}(\varepsilon)=\left\{\varphi \in C_{n}^{+}\right.$: $\varphi_{i}(\theta) \geq \varepsilon$ for $\left.\theta \in[-\tau, 0], i=1, \ldots, n\right\}$ is positively invariant for any $\varepsilon$ with $0<\varepsilon<\min _{i=1, \ldots, n}\left\{\left(\sum_{j=1}^{n} b_{i j} \alpha_{j}\right) / \beta_{i}\right\}$. Lemma 3.2 shows that system (3.1) is point dissipative. Therefore, by Theorem 4.1 .2 of [23], there is a global attractor $G_{\varepsilon}$ in $C_{n}^{+}(\varepsilon)$. Since for a strongly monotone dynamical system, the global attractor contains at least one equilibrium point (see, e.g., Theorem 3.1 of [24]), $G_{\varepsilon}$ contains at least one equilibrium. Therefore, system (3.1) has at least one positive equilibrium state.

We now consider the ordinary differential equations

$$
\dot{x}_{i}=\sum_{j=1}^{n} b_{i j} \alpha_{j} x_{j}-\beta_{i} x_{i}^{2}+\sum_{j \neq i} D_{j i}\left(x_{j}-x_{i}\right), \quad i=1, \ldots, n .
$$

Let $\widetilde{F}=\left(\widetilde{F}_{1}, \ldots, \widetilde{F}_{n}\right): R^{n} \rightarrow R^{n}$ be defined by

$$
\widetilde{F}_{i}=\sum_{j=1}^{n} b_{i j} \alpha_{j} x_{j}-\beta_{i} x_{i}^{2}+\sum_{j \neq i} D_{j i}\left(x_{j}-x_{i}\right), \quad i, \ldots, n .
$$


Obviously, $\frac{\partial \widetilde{F}_{i}}{\partial x_{j}}\left(x_{1}, \ldots, x_{n}\right)=b_{i j} \alpha_{j}+D_{j i} \geq 0$ for $i \neq j, D \widetilde{F}(x)$ is irreducible for $x \in R_{+}^{n}$, since the dispersal matrix $\left(D_{i j}\right)$ is irreducible. Moreover, it is easy to verify that $D \widetilde{F}(y)-D \widetilde{F}(x) \in R_{+}^{n \times n}$ if $x, y \in R_{+}^{n}$ and $x-y \in R_{+}^{n}$. Employing the same argument as that for Lemma 3.1, we can prove that all nonnegative solutions of (3.4) are bounded. Therefore by Lemma 3.4, either there is no positive equilibrium and the origin is globally asymptotically stable, or there is a unique positive equilibrium which is globally asymptotically stable over $R_{+}^{n} \backslash\{0\}$.

On the other hand, we have shown that system (3.1) has at least one positive equilibrium. Clearly, any equilibrium of system (3.1) is also an equilibrium for system (3.4) and conversely. Therefore, (3.4), and hence (3.1), has one and only one positive equilibrium.

This unique positive equilibrium, denoted by $P$, of system (3.1) must be in $C_{n}^{+}(\varepsilon)$ for any $\varepsilon$ with $0<\varepsilon<\min _{i=1, \ldots, n}\left(\sum_{j=1}^{n} b_{i j} \alpha_{j}\right) / \beta_{i}$. By Theorem 3.3 of [25], $\lim _{t \rightarrow \infty} M_{t}(\phi)=P$ for any $\phi \in C_{n}^{+}(\varepsilon)$. This implies that $\lim _{t \rightarrow \infty} M_{t}(\phi)=P$ for any $\phi \in C_{n}^{+} \backslash\{0\}$ since, by Theorem 3.1, for any $\phi \in C_{n}^{+} \backslash\{0\}$ there exists $\varepsilon>0$ such that $M_{t}(\phi) \in C_{n}^{+}(\varepsilon)$ for sufficiently large $t$. The proof is then completed.

We now consider the subsystem for the immature population

$$
\begin{array}{r}
\frac{d}{d t} I_{i}(t)=-\gamma_{i} I_{i}(t)+\sum_{j \neq i} \delta_{j i}\left[I_{j}(t)-I_{i}(t)\right]+\alpha_{i} M_{i}(t)-\sum_{j=1}^{n} b_{i j} \alpha_{j} M_{j}(t-\tau), \\
i=1, \ldots, n .
\end{array}
$$

We have the following global convergence theorem.

THEOREM 3.3. Suppose that the initial mature population $\left(\varphi_{1}, \ldots, \varphi_{n}\right) \in C_{n}^{+}$and

$$
\left(\begin{array}{c}
I_{1}(0) \\
\vdots \\
I_{n}(0)
\end{array}\right) \geq \int_{-\tau}^{0} e^{-A s}\left(\begin{array}{c}
\alpha_{1} \varphi_{1}(s) \\
\vdots \\
\alpha_{n} \varphi_{n}(s)
\end{array}\right) d s
$$

Then $I_{i}(t) \geq 0$ for $t \geq 0$ and $i=1, \ldots, n$. If, in addition, $\left(\varphi_{1}, \ldots, \varphi_{n}\right) \in$ $C_{n}^{+} \backslash\{0\}$, then

$$
\lim _{t \rightarrow \infty}\left(\begin{array}{c}
I_{1}(t) \\
\vdots \\
I_{n}(t)
\end{array}\right)=A^{-1}\left(e^{A \tau}-I\right)\left(\begin{array}{c}
\alpha_{1} M_{1}^{*} \\
\vdots \\
\alpha_{n} M_{n}^{*}
\end{array}\right)
$$

where $\left(M_{1}^{*}, \ldots, M_{n}^{*}\right)$ is the unique positive equilibrium of system (3.1).

Proof. Equation (3.5) can be rewritten in the following vector form

$$
\frac{d}{d t}\left(\begin{array}{c}
I_{1}(t) \\
\vdots \\
I_{n}(t)
\end{array}\right)=A\left(\begin{array}{c}
I_{1}(t) \\
\vdots \\
I_{n}(t)
\end{array}\right)+\left(\begin{array}{c}
\alpha_{1} M_{1}(t) \\
\vdots \\
\alpha_{n} M_{n}(t)
\end{array}\right)-e^{A \tau}\left(\begin{array}{c}
\alpha_{1} M_{1}(t-\tau) \\
\vdots \\
\alpha_{n} M_{n}(t-\tau)
\end{array}\right)
$$


Therefore,

$$
\begin{aligned}
\frac{d}{d t}\left[e^{-A t}\left(\begin{array}{c}
I_{1}(t) \\
\vdots \\
I_{n}(t)
\end{array}\right)\right] & =e^{-A t}\left[\frac{d}{d t}\left(\begin{array}{c}
I_{1}(t) \\
\vdots \\
I_{n}(t)
\end{array}\right)-A\left(\begin{array}{c}
I_{1}(t) \\
\vdots \\
I_{n}(t)
\end{array}\right)\right] \\
& =e^{-A t}\left[\left(\begin{array}{c}
\alpha_{1} M_{1}(t) \\
\vdots \\
\alpha_{n} M_{n}(t)
\end{array}\right)-e^{A \tau}\left(\begin{array}{c}
\alpha_{1} M_{1}(t-\tau) \\
\vdots \\
\alpha_{n} M_{n}(t-\tau)
\end{array}\right)\right] \\
& =\frac{d}{d t} \int_{t-\tau}^{t} e^{-A s}\left(\begin{array}{c}
\alpha_{1} M_{1}(s) \\
\vdots \\
\alpha_{n} M_{n}(s)
\end{array}\right) d s
\end{aligned}
$$

from which it follows that

$$
e^{-A t}\left(\begin{array}{c}
I_{1}(t) \\
\vdots \\
I_{n}(t)
\end{array}\right)-\int_{t-\tau}^{t} e^{-A s}\left(\begin{array}{c}
\alpha_{1} M_{1}(s) \\
\vdots \\
\alpha_{n} M_{n}(s)
\end{array}\right) d s=\left(\begin{array}{c}
I_{1}(0) \\
\vdots \\
I_{n}(0)
\end{array}\right)-\int_{-\tau}^{0} e^{-A s}\left(\begin{array}{c}
\alpha_{1} M_{1}(s) \\
\vdots \\
\alpha_{n} M_{n}(s)
\end{array}\right) d s .
$$

That is,

$$
\left(\begin{array}{c}
I_{1}(t) \\
\vdots \\
I_{n}(t)
\end{array}\right)=e^{A t}\left[\left(\begin{array}{c}
I_{1}(0) \\
\vdots \\
I_{n}(0)
\end{array}\right)-\int_{-\tau}^{0} e^{-A s}\left(\begin{array}{c}
\alpha_{1} \varphi_{1}(s) \\
\vdots \\
\alpha_{n} \varphi_{n}(s)
\end{array}\right) d s\right]+\int_{0}^{\tau} e^{A \theta}\left(\begin{array}{c}
\alpha_{1} M_{1}(t-\theta) \\
\vdots \\
\alpha_{n} M_{n}(t-\theta)
\end{array}\right) d \theta
$$

Therefore our conclusion follows from the fact that $e^{A t}$ is a positive matrix for $t>0, e^{A t} \rightarrow 0$ as $t \rightarrow \infty$,

$$
\begin{gathered}
\left(\begin{array}{c}
\alpha_{1} M_{1}(t) \\
\vdots \\
\alpha_{n} M_{n}(t)
\end{array}\right) \geq\left(\begin{array}{c}
0 \\
\vdots \\
0
\end{array}\right) \quad \text { for } t \geq-\tau, \\
\left(\begin{array}{c}
\alpha_{1} M_{1}(t) \\
\vdots \\
\alpha_{n} M_{n}(t)
\end{array}\right) \rightarrow\left(\begin{array}{c}
\alpha_{1} M_{1}^{*} \\
\vdots \\
\alpha_{n} M_{n}^{*}
\end{array}\right) \quad \text { as } t \rightarrow \infty,
\end{gathered}
$$

and the following relation

$$
\int_{0}^{\tau} e^{A \theta}\left(\begin{array}{c}
\alpha_{1} M_{1}^{*} \\
\vdots \\
\alpha_{n} M_{n}^{*}
\end{array}\right) d \theta=\int_{0}^{\tau} A^{-1} \frac{d}{d \theta} e^{A \theta}\left(\begin{array}{c}
\alpha_{1} M_{1}^{*} \\
\vdots \\
\alpha_{n} M^{*}
\end{array}\right)=A^{-1}\left(e^{A \tau}-I\right)\left(\begin{array}{c}
\alpha_{1} M_{1}^{*} \\
\vdots \\
\alpha_{n} M_{n}^{*}
\end{array}\right) .
$$

REMARK 3.1. To conclude this section, we remark that in the case of symmetric dispersal, i.e., $D_{i j}=0$ iff $\delta_{i j}=0$ and $D_{i j}=0$ iff $D_{j i}=0$ for $i \neq j$, the assumption (H7) can be dropped, since if $D$ or $\Delta$ is reducible, then the subsystem for the mature population in Eq. (2.5) decouples into two or more smaller irreducible subsystems, and consequently our results in this section can be applied to these irreducible subsystems. 
4. Convergence in the case of nonsymmetric dispersion: a two-patch environment. We have considered a single-species population with two life stages which disperses in a multi-patch environment. In the case of symmetric dispersion between different patches, we showed that the subsystem for the mature population can be decoupled into smaller subsystems which are described by cooperative and irreducible retarded functional differential equations, with the time delay representing the time from birth to maturity. By using the monotone dynamical systems theory due to Hirsch and Smith, we proved that there exists one and only one positive equilibrium point which is globally asymptotically stable.

However, the nonsymmetric case is much more complicated even for the model describing a single-species population dispersing in a two-patch environment with $\delta_{21}=D_{21}=0$.

In this case,

$$
A=\left(\begin{array}{cc}
-\gamma_{1} & 0 \\
\delta_{12} & -\gamma_{2}-\delta_{12}
\end{array}\right)
$$

and

$$
e^{A t}=\left(\begin{array}{cc}
e^{-\gamma_{1} t} & 0 \\
e^{-\left(\gamma_{2}+\delta_{12}\right) t} & \int_{0}^{t} e^{-\left(\gamma_{2}+\delta_{12}\right)(t-s)} \delta_{12} e^{-\gamma_{1} s} d s
\end{array}\right)
$$

Therefore,

$$
\left(b_{i j}\right)=\left(\begin{array}{cc}
e^{-\gamma_{1} \tau} & 0 \\
e^{-\left(\gamma_{2}+\delta_{12}\right) \tau} & \int_{0}^{\tau} e^{-\left(\gamma_{2}+\delta_{12}\right)(\tau-s)} \delta_{12} e^{-\gamma_{1} s} d s
\end{array}\right) .
$$

In particular, $b_{12}=0$. Hence the model equation (2.5) becomes

$$
\begin{aligned}
& \frac{d I_{1}(t)}{d t}=-\gamma_{1} I_{1}(t)+\alpha_{1} M_{1}(t)-e^{-\gamma_{1} \tau} \alpha_{1} M_{1}(t-\tau), \\
& \frac{d I_{2}(t)}{d t}=-\gamma_{2} I_{2}(t)+\delta_{12}\left[I_{1}(t)-I_{2}(t)\right]+\alpha_{2} M_{2}(t)-b_{21} \alpha_{1} M_{1}(t-\tau)-b_{22} \alpha_{2} M_{2}(t-\tau), \\
& \frac{d M_{1}(t)}{d t}=-\beta_{1} M_{1}^{2}(t)+e^{-\gamma_{1} \tau} \alpha_{1} M_{1}(t-\tau), \\
& \frac{d M_{2}(t)}{d t}=-\beta_{2} M_{2}^{2}(t)+D_{12}\left[M_{1}(t)-M_{2}(t)\right]+b_{21} \alpha_{1} M_{1}(t-\tau)+b_{22} \alpha_{2} M_{2}(t-\tau) .
\end{aligned}
$$

Because of the nonsymmetric dispersion among different patches, the vector field for the subsystem of the mature population is no longer cooperative and irreducible in the sense of Smith [36]. However, we can employ a similar argument to that of Sec. 3 to prove that for any $\varphi_{1} \in C([-\tau, 0],[0, \infty))$, if $\varphi_{1} \not \equiv 0$, then the solution $M_{1}(t)$ of the following initial value problem

$$
\begin{aligned}
\frac{d}{d t} M_{1}(t) & =\alpha_{1} M_{1}(t-\tau) e^{-\gamma_{1} \tau}-\beta_{1} M_{1}^{2}(t) \\
M_{1}(\theta) & =\varphi_{1}(\theta), \quad \theta \in[-\tau, 0]
\end{aligned}
$$

satisfies $\lim _{t \rightarrow \infty} M_{1}(t)=\left(\alpha_{1} e^{-\gamma_{1} \tau}\right) / \beta_{1}$. 
Applying this result to the fourth equation in system (4.1) we obtain an autonomous system

$$
\begin{aligned}
\frac{d}{d t} m_{2}(t)= & -\beta_{2} m_{2}^{2}(t)+D_{12} \frac{\alpha_{1} e^{-\gamma_{1} \tau}}{\beta_{1}}-D_{12} m_{2}(t) \\
& +b_{21} \alpha_{1} \frac{\alpha_{1} e^{-\gamma_{1} \tau}}{\beta_{1}}+b_{22} \alpha_{2} m_{2}(t-\tau)
\end{aligned}
$$

as a limiting equation of

$$
\begin{aligned}
\frac{d}{d t} M_{2}(t)= & -\beta_{2} M_{2}^{2}(t)+D_{12} M_{1}(t)-D_{12} M_{2}(t) \\
& +b_{21} \alpha_{1} M_{1}(t-\tau)+b_{22} \alpha_{2} M_{2}(t-\tau) .
\end{aligned}
$$

It is easy to show that there exists one and only one positive value $M_{2}^{*}$ such that

$$
-\beta_{2} M_{2}^{*^{2}}+D_{12} \frac{\alpha_{1} e^{-\gamma_{1} \tau}}{\beta_{1}}-D_{12} M_{2}^{*}+b_{21} \alpha_{1} \frac{\alpha_{1} e^{-\gamma_{1} \tau}}{\beta_{1}}+b_{22} \alpha_{2} M_{2}^{*}=0
$$

Evidently, $M_{2}^{*}$ is an equilibrium of (4.3). By using a similar argument to that in Sec. 3, we can prove that $m_{2}(t) \rightarrow M_{2}^{*}$ as $t \rightarrow \infty$ if $m_{2}(\theta) \geq 0$ for $\theta \in[-\tau, 0]$. To prove that $\lim _{t \rightarrow \infty} M_{2}(t)=M_{2}^{*}$ for any solution of (4.4) with $M_{2} \in C_{1}^{+}$, we need the following global convergence theorem via limiting equations, as an extension of a result in [3] to retarded equations.

We consider a nonautonomous retarded equation

$$
\dot{x}(t)=f\left(t, x_{t}\right)
$$

where $f:[0, \infty) \times C\left([-\tau, 0], R^{n}\right) \rightarrow R^{n}$ is continuous. Suppose that $U \subset$ $C\left([-\tau, 0], R^{n}\right)$ is a given closed set such that for any $\varphi \in U$ and $t_{0} \geq 0$, the solution of (4.6) with $x_{t_{0}}=\varphi$ exists uniquely for all $t \geq t_{0}$ and $x_{t}^{f}\left(t_{0}, \varphi\right) \in U$, where $x_{t}^{f}\left(t_{0}, \varphi\right)$ denotes the solution of (4.6) with $x_{t_{0}}^{f}\left(t_{0}, \varphi\right)=\varphi$. We say $f$ is positively precompact with respect to $U$ if, for any sequence $t_{j} \rightarrow \infty$ there exists a subsequence $t_{j_{k}}$ and a continuous functional $g:[0, \infty) \times U \rightarrow R^{n}$ such that $f\left(t_{j_{k}}+t, \varphi\right) \rightarrow g(t, \varphi)$ as $k \rightarrow \infty$ uniformly for $(t, \varphi) \in[0, \infty) \times U$. Let

$$
\begin{aligned}
H(f)=\{ & g:[0, \infty) \times U \rightarrow R^{n}: g \text { is continuous } \\
& \text { and there exists a sequence } t_{k} \rightarrow \infty \text { such that } \\
& \left.f\left(t_{k}+t, \varphi\right) \rightarrow g(t, \varphi) \text { uniformly for }(t, \varphi) \in[0, \infty) \times U\right\} .
\end{aligned}
$$

We say that $f$ is regular if, for any $g \in H(f)$ and $\left(t_{0}, \varphi\right) \in[0, \infty) \times U$, the initial value problem $\dot{x}(t)=g\left(t, x_{t}\right), x_{t_{0}}=\varphi$ has a unique solution, denoted by $x_{t}^{g}\left(t_{0}, \varphi\right)$, defined for all $t \geq t_{0}$ and $x_{t}^{g}\left(t_{0}, \varphi\right) \in U$ for all $t \geq t_{0}$.

THEOREM 4.1. Suppose that $f$ is positively precompact and regular with respect to $U$, and that

(i) there exists a vector $K \in R^{n}$ such that the constant functional $\widehat{K}:[-\tau, 0] \rightarrow$ $R^{n}$ defined by $\widehat{K}(\theta)=K$ for $\theta \in[-\tau, 0]$ belongs to $U$ and $x_{t}^{g}\left(t_{0}, \varphi\right) \rightarrow \widehat{K}$ for $\left(t_{0}, \varphi\right) \in[0, \infty) \times U$ and for every $g \in H(f)$ as $t \rightarrow \infty$; 
(ii) $\widehat{K}$ is eventually uniformly stable with respect to system (4.6) and $U$, namely, for any $\varepsilon>0$ there exists $\delta(\varepsilon)>0$ and $T(\varepsilon)>0$ such that for any $\left(t_{0}, \varphi\right) \epsilon$ $[0, \infty) \times U$, if there exists $t^{*} \geq t_{0}+T(\varepsilon)$ such that $\left\|x_{t^{*}}^{f}\left(t_{0}, \varphi\right)-\widehat{K}\right\| \leq \delta$ then $\left\|x_{t}^{f}\left(t_{0}, \varphi\right)-\widehat{K}\right\| \leq \varepsilon$ for $t \geq t^{*}$.

Then for any $\left(t_{0}, \varphi\right) \in[0, \infty) \times U$ such that $\left\{x_{t}^{f}\left(t_{0}, \varphi\right): t \geq t_{0}\right\}$ has compact closure, it follows that $\lim _{t \rightarrow \infty} x_{t}^{f}\left(t_{0}, \varphi\right)=\widehat{K}$.

Proof. By way of contradiction, if the conclusion is not true, then there exist $\left(t_{0}, \varphi\right) \in[0, \infty) \times U, \varepsilon>0$ and an increasing and unbounded sequence $t_{k} \rightarrow \infty$ as $k \rightarrow \infty$ such that $\left\|x_{t_{k}}^{f}\left(t_{0}, \varphi\right)-\widehat{K}\right\| \geq \varepsilon$ for $k \geq 1$. By assumption (ii), $\| x_{t}^{f}\left(t_{0}, \varphi\right)-$ $\widehat{K} \| \geq \delta(\varepsilon)$ for all $t \geq t_{0}+T(\varepsilon)$. Let $y^{k}(t)=x^{f}\left(t_{k}+t ; t_{0}, \varphi\right), k=1,2, \ldots$. Then $y^{k}(t)$ solves the following initial value problem

$$
\left\{\begin{array}{l}
\dot{y}^{k}(t)=f\left(t_{k}+t, y_{t}^{k}\right), \\
y_{0}^{k}=x_{t_{k}}^{f}\left(t_{0}, \varphi\right) .
\end{array}\right.
$$

Because of the relative compactness of $\left\{x_{t}^{f}\left(t_{0}, \varphi\right) ; t \geq t_{0}\right\}$ and the precompactness of $f$ with respect to $U$, without loss of generality, we may assume that $x_{t_{k}}^{f}\left(t_{0}, \varphi\right) \rightarrow$ $\psi \in U$ and $f\left(t_{k}+t, \xi\right) \rightarrow g(t, \xi)$ as $k \rightarrow \infty$ for $(t, \xi) \in[0, \infty) \times U$, where $g:[0, \infty) \times U \rightarrow R^{n}$ is continuous. Therefore, by the regularity of $f$ and the wellknown continuous dependence of a solution on its initial data and the right-hand side functional of the equation, we obtain that $\lim _{t \rightarrow \infty} y^{k}(t)=x^{g}(t ; 0, \psi)$ uniformly on any compact set in $[0, \infty)$. Let $k$ be sufficiently large such that $t_{k} \geq T(\varepsilon)$. Then $\left\|y_{t}^{k}-\widehat{K}\right\|=\left\|x_{t_{k}+t}^{f}\left(t_{0}, \phi\right)-\widehat{K}\right\| \geq \delta(\varepsilon)$ for $t \geq 0$ from which it follows that $\left\|x_{t}^{g}(0, \psi)-\widehat{K}\right\| \geq \delta(\varepsilon)$ for $t \in[0, \infty)$, contradicting the assumption (i).

Therefore $\lim _{t \rightarrow \infty} x_{t}^{f}\left(t_{0}, \varphi\right)=\widehat{K}$. This completes the proof.

We now apply Theorem 4.1 to system (4.4) and obtain

THEOREM 4.2. For any solution of (4.4) with $M_{2}(\theta) \geq 0$ for $\theta \in[-\tau, 0]$, we have $\lim _{t \rightarrow \infty} M_{2}(t)=M_{2}^{*}$.

Proof. For any solution $M_{1}(t)$ of system (4.2), let $f:[0, \infty) \times C([-\tau, 0], R) \rightarrow R$ be defined by

$$
f(t, \psi)=-\beta_{2} \psi^{2}(0)+b_{22} \alpha_{2} \psi(-\tau)-D_{12} \psi(0)+D_{12} M_{1}(t)+b_{21} \alpha_{1} M_{1}(t-\tau)
$$

for $(t, \psi) \in[0, \infty) \times C([-\tau, 0], R)$. It is easy to prove, in a similar way to the proof of Theorem 3.1, that for any $\varphi \in C_{1}^{+}$and $t_{0} \geq 0$, the solution of (4.4) with $M_{20}=\varphi$ (denoted by $M_{2}^{f}\left(t_{0}, \varphi\right)$ ) and the solution of (4.3) with $m_{20}=\varphi$ (denoted by $M_{2}^{g}\left(t_{0}, \varphi\right)$ ) satisfy that $M_{2 t}^{f}\left(t_{0}, \varphi\right), M_{2 t}^{g}\left(t_{0}, \varphi\right) \in C_{1}^{+}$for $t \geq t_{0}$, where $g: C([-\tau, 0], R) \rightarrow R$ is defined by

$$
g(\psi)=-\beta_{2} \psi^{2}(0)+b_{22} \alpha_{2} \psi(-\tau)-D_{12} \psi(0)+\left(D_{12}+b_{21} \alpha_{1}\right) \frac{e^{\gamma_{1} \tau} \alpha_{1}}{\beta_{1}}
$$

for $\psi \in C([-\tau, 0], R)$. 
Evidently, $f$ is positively precompact and regular with respect to $C_{1}^{+}$, and $H(f)=$ $\{g\}$. Using a similar argument to that in Sec. 3, we can prove that for any $\left(t_{0}, \varphi\right) \in$ $[0, \infty) \times C_{1}^{+}, \lim _{t \rightarrow \infty} M_{2 t}^{g}\left(t_{0}, \varphi\right)=\widehat{M}_{2}^{*}$.

We now prove that $\widehat{M}_{2}^{*}$ is eventually uniformly stable with respect to system (4.4) and $C_{1}^{+}$. Since $\widehat{M}_{2}^{*}$ is globally uniformly asymptotically stable with respect to system (4.3), by the well-known inverse theorem of Liapunov direct method (see, e.g., [43]), there exist a constant $\delta_{0}>0$ and a continuous functional $V(\varphi)$ defined for $\varphi \in C_{1}^{+}$with $\left\|\varphi-\widehat{M}_{2}^{*}\right\| \leq \delta_{0}$, a constant $L>0$ and increasing continuous functions $\alpha, \beta, \gamma:[0, \infty) \rightarrow[0, \infty)$ with $\alpha(0)=\beta(0)=\gamma(0)=0$ such that

$$
\begin{aligned}
\alpha\left(\left\|\varphi-\widehat{M}_{2}^{*}\right\|\right) & \leq V(\varphi) \leq \beta\left(\left\|\varphi-\widehat{M}_{2}^{*}\right\|\right), \\
|V(\varphi)-V(\psi)| & \leq L\|\varphi-\psi\|, \\
\frac{d}{d t} V\left(M_{2 t}^{g}\left(t_{0}, \varphi\right)\right) & \leq-\gamma\left(\left\|M_{2 t}^{g}\left(t_{0}, \varphi\right)-\widehat{M}_{2}^{*}\right\|\right)
\end{aligned}
$$

for all $\varphi, \psi \in C_{1}^{+}$with $\left\|\varphi-\widehat{M}_{2}^{*}\right\| \leq \delta_{0}$ and $\left\|\psi-\widehat{M}_{2}^{*}\right\| \leq \delta_{0}$. Therefore

$$
\frac{d}{d t} V\left(M_{2 t}^{f}\left(t_{0}, \varphi\right)\right) \leq-\gamma\left(\left\|M_{2 t}^{f}\left(t_{0}, \varphi\right)-\widehat{M}_{2}^{*}\right\|\right)+L\left(D_{12}+b_{21} \alpha_{1}\right)\left\|M_{1 t}-\frac{e^{-\gamma \tau} \alpha_{1}}{\beta_{1}}\right\| .
$$

For any $\varepsilon>0$, there exists $\delta(\varepsilon)>0$ and $T(\varepsilon)>0$ such that $\beta(\delta(\varepsilon))<\alpha(\varepsilon)$ and $L\left(D_{12}+b_{21} \alpha_{1}\right)\left\|M_{1 t}-\left(\alpha_{1} \hat{e}^{-\gamma_{1} \tau}\right) / \beta_{1}\right\| \leq \gamma(\delta(\varepsilon))$ for $t \geq t_{0}+T(\varepsilon)$. If at an instant $t^{*} \geq t_{0}+T(\varepsilon),\left\|M_{2 t^{*}}^{f}\left(t_{0}, \varphi\right)-\widehat{M}_{2}^{*}\right\|<\delta(\varepsilon)$, then we claim that $\left\|M_{2 t}^{f}\left(t_{0}, \varphi\right)-\widehat{M}_{2}^{*}\right\|<\varepsilon$ for $t \geq t^{*}$. Otherwise, there exists $t_{2}>t_{1} \geq t^{*}$ such that $\left\|M_{2 t_{1}}^{f}\left(t_{0}, \varphi\right)-\widehat{M}_{2}^{*}\right\|=\delta(\varepsilon)$, $\left\|M_{2 t_{2}}^{f}\left(t_{0}, \varphi\right)-\widehat{M}_{2}^{*}\right\|=\varepsilon$ and $\delta(\varepsilon) \leq\left\|M_{2 t}^{f}\left(t_{0}, \phi\right)-\widehat{M}_{2}^{*}\right\| \leq \varepsilon$ for $t \in\left[t_{1}, t_{2}\right]$. Therefore on $\left[t_{1}, t_{2}\right]$, we have

$$
\frac{d}{d t} V\left(M_{2 t}^{f}\left(t_{0}, \varepsilon\right)\right)=-\gamma(\delta(\varepsilon))+L\left(D_{12}+b_{21} \alpha_{1}\right)\left\|M_{1 t}-\frac{\alpha_{1} \hat{e}^{-\gamma_{1} \tau}}{\beta_{1}}\right\| \leq 0
$$

from which it follows that

$$
\begin{aligned}
\alpha(\varepsilon) & =\alpha\left(\left\|M_{2 t_{2}}^{f}\left(t_{0}, \varphi\right)-\widehat{M}_{2}^{*}\right\|\right) \leq V\left(M_{2 t_{2}}^{f}\left(t_{0}, \varphi\right)\right) \\
& \leq V\left(M_{2 t_{1}}^{f}\left(t_{0}, \varphi\right)\right) \leq \beta\left(\left\|M_{2 t_{1}}^{f}\left(t_{0}, \varphi\right)-\widehat{M}_{2}^{*}\right\|\right) \leq \beta(\delta(\varepsilon))
\end{aligned}
$$

which is contrary to $\beta(\delta(\varepsilon))<\alpha(\varepsilon)$.

Therefore by Theorem 4.1, $\lim _{t \rightarrow \infty} M_{2 t}^{f}\left(t_{0}, \varphi\right)=\widehat{M}_{2}^{*}$ for all $t_{0} \geq 0$ and $\varphi \in C_{1}^{+}$. This proves the theorem.

Hence, we have shown the global asymptotic stability of the positive equilibrium for mature populations. It is easy to establish the global convergence of immature populations.

5. Convergence in the case of nonsymmetric dispersal: a general multi-patch environment. In this section, we briefly indicate how the approaches employed in the previous sections can be effectively combined to study the global asymptotic stability of a positive equilibrium state in a more general nonsymmetric dispersion 
situation where the environment is assumed to consist of several groups (denoted by $G_{1}, G_{2}, \ldots, G_{m}$ ) of patches such that in each group the patches are connected by dispersal and there further exists dispersal from patches in the group $G_{k}$ to the patches in groups $G_{p}, k \leq p \leq m$, but there exists no dispersal from the patches in groups $G_{p}, k \leq p \leq m$, to the patches in group $G_{k}$, where $k=1, \ldots, m$.

To derive a model equation, we suppose that the group $G_{k}, 1 \leq k \leq m$, consists of patches $P_{k 1}, \ldots, P_{k n_{k}}$. The following notations will be used throughout this section.

$\gamma_{k j}$ : the death rate of the immature population in the patch $P_{k j}$;

$\alpha_{k j}$ : the birth rate into the immature population in the patch $P_{k j}$;

$\beta_{k j}$ : the death rate of the mature population in the patch $P_{k j}$;

$I_{k j}:$ the immature population in the patch $P_{k j}$;

$M_{k j}:$ the mature population in the patch $P_{k j}$;

$x_{k j}(s, t-\tau):$ the growth rate at the instant $s, t-\tau \leq s \leq t$, of the immature population in the patch $P_{k j}$ born at the instant $t-\tau$, where $1 \leq k \leq m$ and $1 \leq j \leq n_{k}$

$\delta_{k j h}$ : the dispersal coefficient of immature population from the patch $P_{k h}$ to $P_{k j}$;

$D_{k j h}$ : the dispersal coefficient of mature population from the patch $P_{k h}$ to $P_{k j}$, where $1 \leq k \leq m, 1 \leq j \neq h \leq n_{k}$;

$\delta_{k j}^{q r}$ : the dispersal coefficient of the immature population from the patch $P_{q r}$ to the patch $P_{k j}$;

$D_{k j}^{q r}$ : the dispersal coefficient of the mature population from the patch $P_{q r}$ to the patch $P_{k j}$, where $2 \leq k \leq m, 1 \leq j \leq n_{k}, 1 \leq q<k, 1 \leq r \leq n_{q}$.

Then based on the same argument as in Sec. 2, we have the following model equation

$$
\begin{aligned}
\frac{d}{d t} I_{k j}(t)= & -\gamma_{k j} I_{k j}(t)+\alpha_{k j} M_{k j}(t)-x_{k j}(t, t-\tau) \\
& +\sum_{h \neq j} \delta_{k j h}\left[I_{k h}(t)-I_{k j}(t)\right]+\sum_{\substack{q<k \\
1 \leq r \leq n_{q}}} \delta_{k j}^{q r}\left[I_{q r}(t)-I_{k j}(t)\right], \\
\frac{d}{d t} M_{k j}(t)= & -\beta_{k j} M_{k j}^{2}(t)+x_{k j}(t, t-\tau) \\
& +\sum_{h \neq j} D_{k j h}\left[M_{k h}(t)-M_{k j}(t)\right]+\sum_{\substack{q<k \\
1 \leq r \leq n_{q}}} D_{k j}^{q r}\left[M_{q r}(t)-M_{k j}(t)\right], \\
\frac{\partial}{\partial s} y_{k j}(s, t-\tau)= & x_{k j}(s, t-\tau), \quad x_{k j}(t-\tau, t-\tau)=\alpha_{k j} M_{k j}(t-\tau), \\
\frac{\partial}{\partial s} y_{k j}(s, t-\tau)= & -\gamma_{k j} y_{k j}(s, t-\tau)+\sum_{h \neq j} \delta_{k j h}\left[y_{k h}(s, t-\tau)-y_{k j}(s, t-\tau)\right] \\
& +\sum_{\substack{q<k \\
1 \leq r \leq n_{q}}} \delta_{k j}^{q r}\left[y_{q r}(s, t-\tau)-y_{k j}(s, t-\tau)\right],
\end{aligned}
$$

where $t-\tau \leq s \leq t, 1 \leq k \leq m, 1 \leq j \leq n_{k}$. 
To simplify the above model, we introduce the following notations:

$$
\begin{aligned}
& I_{k}(t)=\left(I_{k 1}(t), \ldots, I_{k n_{k}}(t)\right)^{T}, \\
& M_{k}(t)=\left(M_{k 1}(t), \ldots, M_{k n_{k}}(t)\right)^{T}, \\
& x_{k}(s, t-\tau)=\left(x_{k 1}(s, t-\tau), \ldots, x_{k n_{k}}(s, t-\tau)\right)^{T} \text {, } \\
& y_{k}(s, t-\tau)=\left(y_{k 1}(s, t-\tau), \ldots, y_{k n_{k}}(s, t-\tau)\right)^{T} \text {, } \\
& \alpha_{k} M_{k}(t)=\left(\alpha_{k 1} M_{k 1}(t), \ldots, a_{k n_{k}} M_{k n_{k}}(t)\right)^{T} \text {, } \\
& \beta_{k} M_{k}^{2}(t)=\left(\beta_{k 1} M_{k 1}^{2}(t), \ldots, \beta_{k n_{k}} M_{k n_{k}}^{2}(t)\right)^{T} \text {, } \\
& \Delta_{k}=\left(\delta_{k j h}\right)_{1 \leq j, h \leq n_{k}} \text { with } \delta_{k j j}=0,1 \leq j \leq n_{k} \text {, } \\
& D_{k}=\left(D_{k j h}\right)_{1 \leq j, h \leq n_{k}} \text { with } D_{k j j}=0,1 \leq j \leq n_{k} \text {, } \\
& \Gamma_{k}=\operatorname{diag}\left(\gamma_{k j}+\sum_{h \neq j} \delta_{k j h}+\sum_{\substack{q<k \\
1 \leq r \leq n_{q}}} \delta_{k j}^{q r}\right)_{1 \leq j \leq n_{k}} \text {, } \\
& L_{k}=\operatorname{diag}\left(\sum_{h \neq j} D_{k j h}+\sum_{\substack{q<k \\
1 \leq r \leq n_{q}}} D_{k j}^{q r}\right)_{\substack{1 \leq j \leq n_{k} \\
1 \leq r \leq}}, \\
& \Delta_{k q}=\left(\delta_{k j}^{q r}\right)_{\substack{1 \leq j \leq n_{k} \\
1 \leq r \leq n_{q}}}, \\
& D_{k q}=\left(D_{k j}^{q r}\right)_{\substack{1 \leq j \leq n_{k} \\
1 \leq r \leq n_{q}}},
\end{aligned}
$$

where $1 \leq k \leq m$. Then the model equation can be rewritten in the following vector form

$$
\begin{aligned}
\frac{d}{d t} I_{k}(t) & =-\Gamma_{k} I_{k}(t)+\alpha_{k} M_{k}(t)-x_{k}(t, t-\tau)+\Delta_{k} I_{k}(t)+\sum_{q<k} \Delta_{k q} I_{q}(t), \\
\frac{d}{d t} M_{k}(t) & =-\beta_{k} M_{k}^{2}(t)+x_{k}(t, t-\tau)+D_{k} M_{k}(t)-L_{k} M_{k}(t)+\sum_{q<k} D_{k q} M_{q}(t), \\
x_{k}(t-\tau, t-\tau) & =\alpha_{k} M_{k}(t-\tau), \\
\frac{\partial}{\partial s} y_{k}(s, t-\tau) n & =x_{k}(s, t-\tau), \quad t-\tau \leq s \leq t, \\
\frac{\partial}{\partial s} y_{k}(s, t-\tau) & =-\Gamma_{k} y_{k}(s, t-\tau)+\Delta_{k} y_{k}(s, t-\tau)+\sum_{q<k} \Delta_{k q} y_{q}(s, t-\tau) .
\end{aligned}
$$

Let

$$
A=\left(\begin{array}{cccc}
-\Gamma_{1}+\Delta_{1} & 0 & \cdots & 0 \\
\Delta_{21} & -\Gamma_{2}+\Delta_{2} & \cdots & 0 \\
& \cdots & & \\
\Delta_{m 1} & \Delta_{m 2} & \cdots & -\Gamma_{m}+\Delta_{m}
\end{array}\right) .
$$


By the assumption that patches in the same group are connected by dispersion, $\Delta_{k}$ and $D_{k}$ are irreducible matrix for $1 \leq k \leq m$. This implies that $-\Gamma_{k}+\Delta_{k}$ is an irreducible matrix for $1 \leq k \leq m$, and thus $B_{k k}=e^{\left(-\Gamma_{k}+\Delta_{k}\right) \tau}$ is a positive matrix for $1 \leq k \leq m$.

Using the same approach as in Sec. 2, we obtain the following formula

$$
\left(\begin{array}{c}
x_{1}(t, t-\tau) \\
\vdots \\
x_{m}(t, t-\tau)
\end{array}\right)=\left(\begin{array}{cccc}
B_{11} & 0 & \cdots & 0 \\
B_{21} & B_{22} & \cdots & 0 \\
& \cdots & \cdots & \\
B_{m 1} & B_{m 2} & \cdots & B_{m m}
\end{array}\right)\left(\begin{array}{c}
\alpha_{1} M_{1}(t-\tau) \\
\vdots \\
\alpha_{m} M_{m}(t-\tau)
\end{array}\right)
$$

where

$$
\left(\begin{array}{cccc}
B_{11} & 0 & \cdots & 0 \\
B_{21} & B_{22} & \cdots & \cdots \\
& \cdots & \cdots & \\
B_{m 1} & B_{m 2} & \cdots & B_{m m}
\end{array}\right)=e^{A \tau} .
$$

Because the off diagonal elements of $A$ are nonnegative, each $B_{i j}, 1 \leq j \leq i \leq m$, is nonnegative.

Substituting formula (5.3) into system (5.2), we get the following simplified model equation

$$
\begin{aligned}
\frac{d I_{k}(t)}{d t}= & -\Gamma_{k} I_{k}(t)+\alpha_{k} M_{k}(t)+\Delta_{k} I_{k}(t)+\sum_{q<k} \Delta_{k q} I_{q}(t) \\
& -\sum_{q \leq k} B_{k q} \alpha_{q} M_{q}(t-\tau), \\
\frac{d M_{k}(t)}{d t}= & -B_{k} M_{k}^{2}(t)+B_{k k} \alpha_{k} M_{k}(t-\tau)-L_{k} M_{k}(t)+D_{k} M_{k}(t) \\
& +\sum_{q<k} B_{k q} \alpha_{q} M_{q}(t-\tau)+\sum_{q<k} D_{k q} M_{q}(t),
\end{aligned}
$$

where $1 \leq k \leq m$.

The first equation for the mature population turns out to be

$$
\frac{d M_{1}(t)}{d t}=-\beta_{1} M_{1}^{2}(t)-L_{1} M_{1}(t)+B_{11} \alpha_{1} M_{1}(t-\tau)+D_{1} M_{1}(t) .
$$

Since $B_{11}$ is a positive matrix and $D_{1}$ is irreducible, we can use the approach in Sec. 3 to prove that there exists a unique positive vector $M_{1}^{*}$ in $R^{n_{1}}$ such that $\lim _{t \rightarrow \infty} M_{1}(t)=M_{1}^{*}$ if $M_{1}(\theta) \geq 0$ for $\theta \in[-\tau, 0]$ and $M_{1} \not \equiv 0$ on $[-\tau, 0]$.

Therefore, the system

$$
\begin{aligned}
\frac{d m_{2}(t)}{d t}= & -\beta_{2} m_{2}^{2}(t)+B_{22} \alpha_{2} m_{2}(t-\tau)-L_{2} m_{2}(t)+D_{2} m_{2}(t) \\
& +B_{21} \alpha_{1} M_{1}^{*}+D_{21} M_{1}^{*}
\end{aligned}
$$

is a limiting equation of the second equation for the mature population

$$
\begin{aligned}
\frac{d M_{2}(t)}{d t}= & -\beta_{2} M_{2}^{2}(t)+B_{22} \alpha_{2} M_{2}(t)-L_{2} M_{2}(t)+D_{2} M_{2}(t) \\
& +B_{21} \alpha_{1} M_{1}(t-\tau)+D_{21} M_{1}(t) .
\end{aligned}
$$


Using the same argument used in Sec. 3, we can prove that there exists a positive vector $M_{2}^{*}$ in $R^{n_{2}}$ such that $\lim _{t \rightarrow \infty} m_{2}(t)=M_{2}^{*}$ if $m_{2}(\theta) \geq 0$ for $\theta \in[-\tau, 0]$. Therefore, employing the argument of Sec. 4, we can prove that $\lim _{t \rightarrow \infty} M_{2}(t)=M_{2}^{*}$ provided $M_{2}(\theta) \geq 0$ for $\theta \in[-\tau, 0]$.

Repeating the above argument for a finite number of steps $(m)$, we obtain that there exists a positive vector $\left(M_{1}^{*}, \ldots, M_{m}^{*}\right) \in R^{n_{1}+\cdots+n_{m}}$ such that

$$
\lim _{t \rightarrow \infty}\left(M_{1}(t), \ldots, M_{m}(t)\right)=\left(M_{1}^{*}, \ldots, M_{m}^{*}\right)
$$

if $M_{i}(\theta) \geq 0$ for $\theta \in[-\gamma, 0]$ and $i=1, \ldots, m$ and $M_{1} \not \equiv 0$ on $[-\tau, 0]$.

Finally, using the same argument as for Theorem 3.3, we obtain the following global convergence theorem.

THEOREM 5.1. There exists a positive vector $\left(M_{1}^{*}, \ldots, M_{m}^{*}\right) \in R^{n_{1}+\cdots+n_{m}}$ such that if

(i) $M_{k}(\theta) \geq 0$ for $\theta \in[-\tau, 0]$ and $k=1, \ldots, m, M_{1} \neq 0$ on $[-\tau, 0]$,

$$
\left(\begin{array}{c}
I_{1}(0) \\
\vdots \\
I_{m}(0)
\end{array}\right) \geq \int_{-\tau}^{0} e^{-A s}\left(\begin{array}{c}
\alpha_{1} M_{1}(\theta) \\
\vdots \\
\alpha_{m} M_{m}(\theta)
\end{array}\right) d \theta
$$

then

$$
\begin{gathered}
\lim _{t \rightarrow \infty}\left(M_{1}(t), \ldots, M_{m}(t)\right)=\left(M_{1}^{*}, \ldots, M_{m}^{*}\right), \\
\lim _{t \rightarrow \infty}\left(I_{1}(t), \ldots, I_{m}(t)\right)=A^{-1}\left(e^{A \tau}-I\right)\left(\begin{array}{c}
\alpha_{1} M_{1}^{*} \\
\vdots \\
\alpha_{m} M_{m}^{*}
\end{array}\right) .
\end{gathered}
$$

REMARK 5.1. The above model can be thought of as describing stage structured population dispersal in a patchy environment where each patch is subdivided into subpatches.

REMARK 5.2. It would be interesting to consider the competition of the mature and immature individuals for resources. We wish to investigate such a competition in a further paper.

\section{REFERENCES}

[1] W. G. Aiello and H. I. Freedman, A time-delay model of single-species growth with stage structure, Math. Biosci. 101, 139-153 (1990)

[2] F. S. Anderson, Competition in populations of one age group, Biometrica 16, 19-27 (1960)

[3] Z. Artstein, Uniform asymptotic stability via the limiting equations, J. Differential Equations 27, 172-189 (1978)

[4] H. J. Barclay and P. Van den Driessche, A model for a species with two life history stages and added mortality, Ecol. Model 11, 157-166 (1980)

[5] E. Beretta, F. Solimano, and Y. Takeuchi, Global stability and periodic orbits for two-patch predatorprey diffusion-delay models, Math. Biosci. 85, 153-183 (1987)

[6] E. Beretta and Y. Takeuchi, Global stability of single-species diffusion models with continuous time delays, Bull. Math. Biol. 49, 431-448 (1987)

[7] G. J. Butler, H. I. Freedman, and P. Waltman, Uniformly persistent systems, Proc. Amer. Math. Soc. 96, 425-430 (1986) 
[8] G. J. Butler and P. Waltman, Persistence in dynamical systems, J. Differential Equations 63, 255$263(1986)$

[9] H. I. Freedman, Single species migration in two habitats: persistence and extinction, Math. Model 8, 778-780 (1987)

[10] H. I. Freedman, Persistence and extinction in models of two-habitat migration, Math. Comput. Modelling 12 105-112 (1989)

[11] H. I. Freedman, B. Rai, and P. Waltman, Mathematical models of population interactions with dispersal II: differential survival in a change of habitat, J. Math. Anal. Appl. 115, 140-154 (1986)

[12] H. I. Freedman, J. B. Shukla, and Y. Takeuchi, Population diffusion in a two-patch environment, Math. Biosci. 95, 111-123 (1989)

[13] H. I. Freedman and Y. Takeuchi, Global stability and predator dynamics in a model of prey dispersal in a patchy environment, Nonlinear Anal. 13, 993-1002 (1989)

[14] H. I. Freedman and Y. Takeuchi, Predator survival versus extinction as a function of dispersal in a predator-prey model with patchy environment, Appl. Anal. 31, 247-266 (1989)

[15] H. I. Freedman and P. Waltman, Mathematical models of population interaction with dispersal I: Stability of two habitats with and without a predator, SIAM J. Appl. Math. 32, 631-648 (1977)

[16] H. I. Freedman and J. H. Wu, Steady state analysis in a model for population diffusion in a multi-patch environment, preprint

[17] W. S. C. Gurney and R. M. Nisbet, Fluctuating periodicity, generation separation, and the expression of larval competition, Theor. Pop. Biol. 28, 150-180 (1985)

[18] W. S. C. Gurney, R. M. Nisbet, and J. H. Lawton, The systematic formulation of tractable single species population models incorporating age structure, J. Animal Ecol. 52, 479-495 (1983)

[19] J. R. Haddock, T. Krisztin, and J. H. Wu, Asymptotic equivalence of neutral equations and retarded equations with infinite delay, Nonlinear Anal. 14, 369-377 (1990)

[20] J. R. Haddock and J. Terjeki, Liapunov-Razumikhin functions and invariance principle for functional differential equations, J. Differential Equations 48, 95-122 (1983)

[21] J. K. Hale, Theory of functional Differential Equations, Springer-Verlag, New York, 1979

[22] J. K. Hale, Asymptotic Behavior of Dissipative Systems, Mathematical Surveys and Monographs, Vol. 25, Amer. Math. Soc., Providence, 1988

[23] H. Hastings, Dynamics of a single species in a spatially varying environment: the stabilizing role of high dispersal rates, J. Math. Biol. 16, 49-55 (1982)

[24] M. W. Hirsch, The dynamical systems approach to differential equations, Bull. Amer. Math. Soc. 11, 1-64 (1984)

[25] R. D. Holt, Population dynamics in two patch environment: some anomalous consequences of optional habitat selection, Theor. Pop. Biol. 28, 181-208 (1985)

[26] Yu. S. Koslesov, Properties of solutions of a class of equations with lag which describe the dynamics of change in the population of a species with the age structure taken into account, Math. USSR Sb. 45, 91-100 (1983)

[27] V. Lakshmikantham and S. Leela, Differential and Integral Inequalities, Vol. 2, Academic Press, New York, 1969

[28] H. D. Landahl and B. D. Hanson, A three stage population model with cannibalism, Bull. Math. Biol. 37, 11-17 (1975)

[29] S. A. Levin, Dispersion and population interactions, Am. Nat. 108, 207-228 (1974)

[30] R. H. Martin and H. L. Smith, Reaction-diffusion systems with time delays: monotonicity invariance, comparison and convergence, preprint

[31] S. W. Pacala and J. Roughgarden, Spatial heterogeneity and interspecific competition, Theor. Pop. Biol. 21, 92-113 (1982)

[32] G. R. Sell, Nonautonomous differential equations and topological dynamics, I. The basic theory, Trans. Amer. Math. Soc. 127, 241-262 (1967)

[33] G. R. Sell, Nonautonomous differential equations and topological dynamics, II. Limiting equations, Trans. Amer. Math. Soc. 127, 263-283 (1967)

[34] N. Shigesada and J. Roughgarden, The role of rapid dispersal in the population dynamics of competition, Theor. Pop. Biol. 21, 353-372 (1982)

[35] J. G. Skellam, Random dispersal in theoretical populations, Biometrika 38, 196-218 (1951)

[36] H. L. Smith, Monotone semiflows generated by functional differential equations, J. Differential Equations 66, 420-442 (1987)

[37] Y. Takeuchi, Global stability in generalized Lotka-Volterra diffiusion systems, J. Math. Anal. Appl. 116, 209-221 (1986) 
[38] Y. Takeuchi, Diffusion effect on stability of Lotka-Volterra models, Bull. Math. Biol. 48, 585-601 (1986)

[39] R. R. Vance, The effect of dispersal on population stability in one-species, discrete-space population growth models, Am. Nat. 123, 230-254 (1984)

[40] G. F. Webb, Theory of Nonlinear Age-Dependent Population Dynamics, Marcel Dekker, New York, 1985

[41] S. N. Wood, S. P. Blythe, S. C. Gurney, and R. M. Nisbet, Instability in mortality estimation schemes related to stage-structure population models, IMA J. Math. Appl. Med. Biol. 6, 47-68 (1989)

[42] T. Yoshizawa, Stability Theory by Liapunov's Second Method, Publications of the Mathematical Society of Japan, No. 9, The Mathematical Society of Japan, Tokyo, 1966 